\title{
THE PARTITION OF ENERGY AMONG SEISMIC WAVES REFLECTED AND REFRACTED AT THE EARTH'S CORE*
}

\author{
By Stephen W. Dana
}

The Reflection and refraction of seismic waves has been studied extensively in the past by various authors. Among them are $\operatorname{Knott}(9,10),{ }^{1}$ Wiechert (13), Zoeppritz (14), Jeffreys (8), Bouasse (3), Gutenberg (4, 5, 6, 7), Berlage (1), Blut (2), and Macelwane $(11,12)$. The purpose of the present paper is to calculate the ratios of the energies of waves reflected and refracted at the surface of the earth's core for various angles of incidence, supposing values for the elastic constants and densities considered the most likely.

Knott (10) in 1919 used similar calculations but different equations to determine the energies of the reflected and refracted waves in terms of the energy of the incident wave, where the incident wave is in the core against the mantle or in the mantle against the core. He assumed the limiting case of an earth consisting of an elastic shell which passes abruptly into a nonrigid elastic core of the same compressibility and density. That is, Knott's assumption was that as the core is approached the material of the earth changes abruptly from an elastic solid to an elastic, greatly compressed liquid. Essentially the same assumption is made in this work except that the density of the core is taken as almost twice that of the surrounding mantle.

The notation used is given in the following table.P designates a longitudinal wave and $\mathrm{S}$ a transverse or shear wave. Two kinds of shear waves will be referred to, the $\mathrm{SV}$ type (direction of vibration in the plane of incidence) and the SH type (direction of vibration perpendicular to the plane of incidence). $\rho$ is the symbol for density:

\begin{tabular}{|c|c|c|c|}
\hline Wave & Velocity & Amplitude & $\begin{array}{l}\text { Angle with normal } \\
\text { to discontinuity }\end{array}$ \\
\hline Incident $\mathrm{P}$. & $v$ & $A$ & $a$ \\
\hline Incident S. . & $w$ & $B$ & $b$ \\
\hline Reflected P.. & $v$ & $C$ & $c$ \\
\hline Reflected S. & $w$ & $D$ & $d$ \\
\hline Refracted P. & $V$ & $E$ & $e$ \\
\hline Refracted S............. & $W$ & $F$ & $f$ \\
\hline
\end{tabular}

$q$ will denote the density ratio $\rho_{2} / \rho_{1}$, where $\rho_{1}$ is the density of the medium containing the incident wave and $\rho_{2}$ is the density of the medium containing the refracted waves.

Suppose then a longitudinal or transverse wave is incident in medium 1 against medium 2, where the media have different elastic properties. Continuity would make it necessary for the normal and tangential strains and stresses to be equal respectively on the two sides of the discontinuity between the two media. For the general case, therefore, six boundary conditions are produced. Equations describing these conditions in terms of the amplitudes and angles of reflection and refraction of the various reflected and refracted waves produced at the discontinuity were obtained by Zoeppritz (14). They may be studied in his original paper or in the work of Gutenberg $(4,5)$ and Macelwane (11, 12), and hence are given below without

\footnotetext{
* Condensed from a thesis submitted in partial fulfillment of the requirements for the degree of Doctor of Philosophy at the California Institute of Technology, Pasadena, California. Manuscript received for publication June 2, 1944.

${ }^{1}$ Numbers in parentheses refer to the references listed at the end of this paper.
} 
derivation. The same assumptions used in their derivation would apply in the following work.

In the general case of an incident longitudinal wave in medium 1 against medium 2, the Zoeppritz equations are:

[1a] Normal strains, $A \cos a-C \cos a+D \sin b-E \cos e-F \sin f=0$.

[1b] Tangential strains, $A \sin a+C \sin a+D \cos b-E \sin e+F \cos f=0$.

[1c] Normal stresses, $-A \cos 2 b-C \cos 2 b+D \frac{w}{v} \sin 2 b+E q \frac{V}{v} \cos 2 f$

$$
+F q \frac{W}{v} \sin 2 f=0 \text {. }
$$

[1d] Tangential stresses, $-A \sin 2 a+C \sin 2 a+D \frac{v}{w} \cos 2 b+E q\left(\frac{W}{w}\right)^{2} \frac{v}{V} \sin 2 e$

$$
-F q\left(\frac{W}{w}\right)^{2} \frac{v}{W} \cos 2 f=0 .
$$

In the general case of an incident shear wave (SV type) in medium 1 against medium 2, the Zoeppritz equations are:

[2a] Normal strains, $-B \sin b-C \cos a+D \sin b-E \cos e-F \sin f=0$.

[2b] Tangential strains, $B \cos b+C \sin a+D \cos b-E \sin e+F \cos f=0$.

[2c] Normal stresses, $B \sin 2 b-C \frac{v}{w} \cos 2 b+D \sin 2 b+E q \frac{V}{w} \cos 2 f$

$$
+F q \frac{W}{w} \sin 2 f=0 \text {. }
$$

[2d] Tangential stresses, $-B \cos 2 b+C \frac{w}{v^{\prime}} \sin 2 a+D \cos 2 b+E q \frac{W^{2}}{w V} \sin 2 e$

$$
-F q \frac{W}{w} \cos 2 f=0 .
$$

In the general case of an incident shear wave (SH type) in medium 1 against medium 2, the Zoeppritz equations are:

[3a] Tangential strains, $B+D-F=0$.

[3b] Tangential stresses, $B-D-q \frac{W \cos f}{w \cos b} F=0$.

The important assumption at the base of the following work is that the core of the earth does not transmit shear waves. This is in accord with the best measurements from seismograms but is not meant to imply that the core is fluid. The assumption made is that the core, though not necessarily liquid, at least resembles a fluid in not transmitting transverse waves. As a consequence of this condition only four specific cases have to be considered: first, that of an incident $\mathbf{P}$ in the mantle against the core; second, that of an incident $\mathrm{P}$ in the core against the mantle; third, that of an incident $\mathrm{SV}$ in the mantle against the core; and fourth, that of an incident SH in the mantle against the core.

The density of the core just within the boundary is taken as $10.1 \mathrm{gm}$. per cc., and the density of the mantle in the vicinity of the core is taken as $5.4 \mathrm{gm}$. per cc. These 
values are from table 63 on p. 341 of Internal Constitution of the Earth (7). The velocity of shear waves in the mantle is taken as $7.25 \mathrm{~km}$. per sec., and that of longitudinal waves as $13.7 \mathrm{~km}$. per sec. These are from table 37 on p. 134 of "On Seismic Waves" (Fourth Paper) (6). The remaining constant, the velocity of $\mathrm{P}$ waves within the boundary of the core, is $8.0 \mathrm{~km}$. per sec. and is taken from $\mathrm{p}$. 278 of (7).

For Case 1 , the incident $\mathrm{P}$ in the mantle against the core, the initial conditions are:

$$
\begin{array}{lll}
W=0 \text { and } \sin f=0 & v=13.7 \mathrm{~km} . \text { per sec. } & \rho_{1}=5.4 \mathrm{gm} . \text { per cc. } \\
w=7.25 \mathrm{~km} . \text { per sec. } & V=8.0 \mathrm{~km} . \text { per sec. } & \rho_{2}=10.1 \mathrm{gm} . \text { per cc. }
\end{array}
$$

and the tangential strains equation disappears. The Zoeppritz equations become:

[4a] Normal strains, $A \cos a-C \cos a+D \sin b-E \cos e=0$.

[4b] Normal stresses, $-A \cos 2 b-C \cos 2 b+D \frac{w}{v} \sin 2 b+E q \frac{V}{v}=0$.

[4c] Tangential stresses, $-A \sin 2 a+C \sin 2 a+D \frac{v}{w} \cos 2 b=0$.

Letting $A=1.00$ and substituting the values of the densities and the velocities,

[5a] Normal strains, $(-\cos a) C+(\sin b) D-(\cos e) E=-\cos a$.

[5b] Normal stresses, $(-\cos 2 b) C+(0.5292 \sin 2 b) D+1.092 E=\cos 2 b$.

[5c] Tangential stresses, $(\sin 2 a) C+(1.8896 \cos 2 b) D=\sin 2 a$.

These are homogeneous linear equations with three unknowns and may therefore be solved by means of the ordinary determinant method. The values of the ratios $C / A, D / A$, and $E / A$ were calculated for these angles of incidence $a: 0^{\circ}, 10^{\circ}, 20^{\circ}, 30^{\circ}$, $40^{\circ}, 50^{\circ}, 60^{\circ}, 70^{\circ}, 80^{\circ}, 83^{\circ}, 84^{\circ}, 85^{\circ}, 89^{\circ}, 90^{\circ}$. The corresponding values of the angles $b$ and $e$ were determined from Snell's law,

$$
\frac{\sin a}{v}=\frac{\sin b}{w}=\frac{\sin c}{v}=\frac{\sin d}{w}=\frac{\sin e}{V}=\frac{\sin f}{W},
$$

which is also used in similar calculations for the other three cases of reflection and refraction under consideration.

The next step was to make use of the work of Blut (2) concerning the distribution of the incident energy among the waves generated at a discontinuity between media of differing elastic properties. As given on page 178 of Macelwane (12), Blut's equation for the energy distribution when the incident wave is longitudinal is

$$
1=\frac{C^{2}}{A^{2}}+\frac{D^{2} \sin 2 b}{A^{2} \sin 2 a}+\frac{E^{2}}{A^{2}} q \frac{\sin 2 e}{\sin 2 a}+\frac{F^{2}}{A^{2}} q \frac{\sin 2 f}{\sin 2 a} .
$$

The assumptions made in its derivation naturally are valid here. Since there is no refracted shear wave in Case $\mathrm{I}, F=0$. The expressions for the values of the relative energy going into the reflected and refracted waves are therefore:

[8a] $\frac{C^{2}}{A^{2}}=$ relative energy of the reflected longitudinal wave. 
$[8 b]$

$\frac{D^{2} \sin 2 b}{A^{2} \sin 2 a}=$ relative energy of the reflected shear wave.

[8c] $\frac{E^{2}}{A^{2}} q \frac{\sin 2 e}{\sin 2 a}=$ relative energy of the refracted longitudinal wave.

These equations, together with the amplitude ratios calculated earlier, were used to find the square roots of the relative energy going into the refracted and reflected

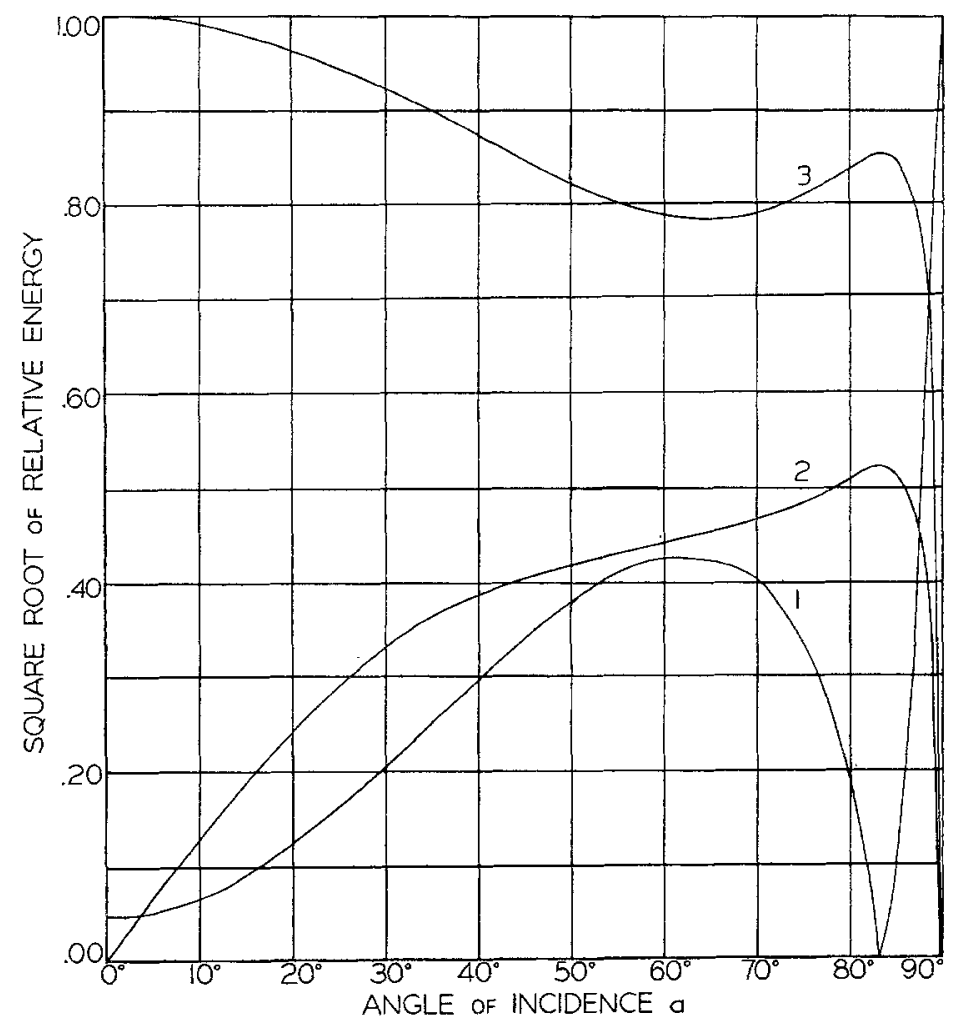

Fig. 1. Incident longitudinal wave in the mantle against the core.

$1=$ square root of the relative energy of the reflected longitudinal wave.

$2=$ square root of the relative energy of the reflected shear wave.

$3=$ square root of the relative energy of the refracted longitudinal wave.

waves at the various values of $a$ between $0^{\circ}$ and $90^{\circ}$. The square roots of relative energy for Case I are plotted against the angle of incidence $a$ in figure 1 . It should be noted that equation [7] gives an excellent check on the calculations using the Zoeppritz equations. Knott (10) also considered the case of an incident longitudinal wave in the mantle against the core. The graph of the relative energies of the reflected and refracted waves plotted against angle of incidence on page 189 of his article is very similar to figure 1 of this paper. The slight differences are due to the different values used for the densities and wave velocities. Knott took $12.89 \mathrm{~km}$. per sec. for $v, 10.15 \mathrm{~km}$. per sec. for $V, 6.88 \mathrm{~km}$. per sec. for $w$, and $\rho_{1}=\rho_{2}$. 
For Case II, the incident $\mathrm{P}$ in the core against the mantle, the initial conditions are:

$$
\begin{array}{lll}
w=0 \text { and } \sin b=0 & v=8.0 \mathrm{~km} \text {. per sec. } & \rho_{1}=10.1 \mathrm{gm} . \text { per cc. } \\
W=7.25 \mathrm{~km} . \text { per sec. } & V=13.7 \mathrm{~km} \text {. per sec. } & \rho_{2}=5.4 \mathrm{gm} \text {. per cc. }
\end{array}
$$

and the tangential strains equation disappears. The Zoeppritz equations become:

[9a] Normal strains, $A \cos a-C \cos a-E \cos e-F \sin f=0$.

[9b] Normal stresses, $-A-C+E q \frac{V}{v} \cos 2 f+F q \frac{W}{v} \sin 2 f=0$.

[9c] Tangential stresses, $E W \sin 2 e-F V \cos 2 f=0$.

Letting $A=1.00$ and substituting the values of the densities and the velocities,

[10a] Normal strains, $(-\cos a) C+(-\cos e) E+(-\sin f) F=-\cos a$.

[10b] Normal stresses, $(-1) C+(0.91558 \cos 2 f) E+(0.48453 \sin 2 f) F=1.00$.

[10e] Tangential stresses, $(7.25 \sin 2 e) E+(-13.7 \cos 2 f) F=0$.

These equations were solved for the ratios $C / A, F / A$, and $E / A$ for the following values of the angle of incidence $a: 0^{\circ}, 10^{\circ}, 20^{\circ}, 30^{\circ}, 33^{\circ}, 35^{\circ}, 35^{\circ} 44^{\prime}, 37^{\circ}, 40^{\circ}, 50^{\circ}, 60^{\circ}$, $70^{\circ}, 80^{\circ}, 90^{\circ}$. It should be noted that beyond $35^{\circ} 44^{\prime}$ the ratio $E / A$ does not exist and that $C / A$ and $F / A$ become complex. The absolute values of the last two are used in the relative energy calculations.

$D=0$ in equation [7], and the expressions for the relative energies in Case II are:

[11a] $\frac{C^{2}}{A^{2}}=$ relative energy of the reflected longitudinal wave.

[11b] $\frac{E^{2}}{A^{2}} q \frac{\sin 2 e}{\sin 2 a}=$ relative energy of the refracted longitudinal wave.

[11c] $\frac{F^{2}}{A^{2}} q \frac{\sin 2 f}{\sin 2 a}=$ relative energy of the refracted shear wave.

The square roots of the relative energy going into the reflected and refracted waves were calculated for the stated values of $a$. A plot of them against angle of incidence is shown in figure 2. As in Case I, the fact that the sum of the relative energies must equal one was used to check the calculations in Case II. Also, figure 2 is quite like the graph on page 189 of Knott's article (10) in which he plotted the relative energies of the reflected and refracted waves against angle of incidence for the case of an incident longitudinal wave in the core against the mantle. The slight differences are due, as in Case I, to the different values which Knott used for the densities and wave velocities.

There is one critical angle of incidence in figure 2 of this paper at $a=35^{\circ} 44^{\prime}$. At this angle the relative energy of the refracted $P$ and $S$ waves becomes zero, and the relative energy of the reflected $\mathrm{P}$ wave becomes one. The reason for this may be demonstrated easily from equations [10a], [10b], and [10c]. $e=90^{\circ}$ at $a=35^{\circ} 44^{\prime}$. Substitution of this gives $F=0$ in [10c]. Then $C=1.00$ results from [10a]. These 
values cause $E$ to equal $\frac{2}{0.91558 \cos 2 f}$ in [10b]. However, since $e=90^{\circ}$, the relative energy of the refracted longitudinal wave becomes zero in equation [11b]. $F=0$ gives zero for the relative energy of the refracted shear wave in equation [11c], and $C=1.00$ gives one for the relative energy of the reflected longitudinal wave in equation [11a].

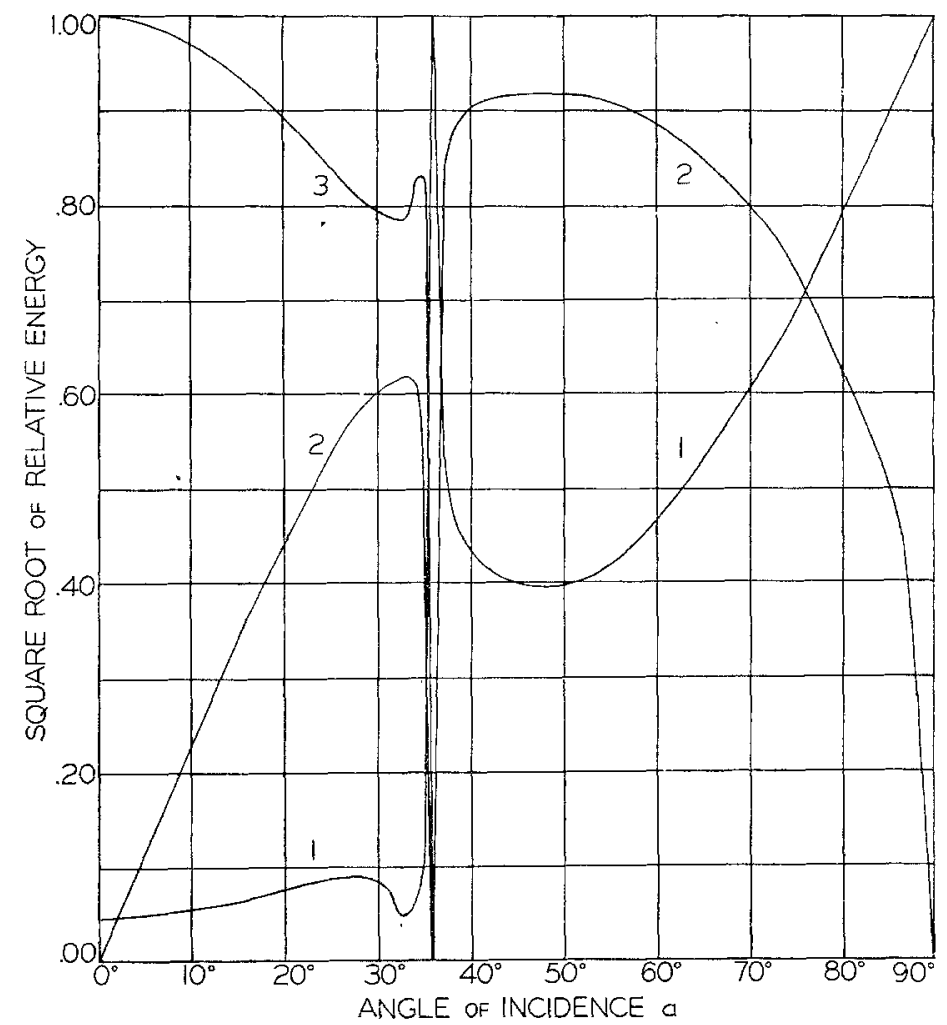

Fig. 2. Incident longitudinal wave in the core against the mantle.

$1=$ square root of the relative energy of the reflected longitudinal wave.

$2=$ square root of the relative energy of the refracted shear wave.

$3=$ square root of the relative energy of the refracted longitudinal wave.

For Case III, the incident SV in the mantle against the core, the initial conditions are:
$W=0$ and $\sin f=0$
$v=13.7 \mathrm{~km}$. per sec.
$\rho_{1}=5.4$ gm. per cc.
$w=7.25 \mathrm{~km}$. per sec.
$V=8.0 \mathrm{~km}$. per sec.
$\rho_{2}=10.1 \mathrm{gm}$. per cc.

and the tangential strains equation disappears. The Zoeppritz equations become:

[12a] Normal strains, $-B \sin b-C \cos a+D \sin b-E \cos e=0$.

[12b] Normal stresses, $B \sin 2 b-C \frac{v}{w} \cos 2 b+D \sin 2 b+E q \frac{V}{w}=0$.

[12c] Tangential stresses, $-B \cos 2 b+\frac{w}{v} \sin 2 a+D \cos 2 b=0$. 
Letting $B=1.00$ and substituting the values of the densities and the velocities,

[13a] Normal strains, $(-\cos a) C+(\sin b) D+(-\cos e) E=\sin b$.

[13b] Normal stresses, $(-1.8897 \cos 2 b) C+(\sin 2 b) D+(2.0639) E=-\sin 2 b$.

[13c] Tangential stresses, $(0.5292 \sin 2 a) C+(\cos 2 b) D=\cos 2 b$.

The ratios $C / B, D / B$, and $E / B$ were calculated from these equations for the following values of the angle of incidence $b: 0^{\circ}, 10^{\circ} 23^{\prime}, 21^{\circ} 47^{\prime}, 30^{\circ} 00^{\prime}, 31^{\circ} 00^{\prime}, 31^{\circ} 57^{\prime} 06^{\prime \prime}$, $33^{\circ} 00^{\prime}, 40^{\circ} 00^{\prime}, 50^{\circ} 00^{\prime}, 60^{\circ} 00^{\prime}, 64^{\circ} 00^{\prime}, 64^{\circ} 59^{\prime} 30^{\prime \prime}, 66^{\circ} 00^{\prime}, 70^{\circ} 00^{\prime}, 80^{\circ} 00^{\prime}, 90^{\circ} 00^{\prime}$. If $b=31^{\circ} 57^{\prime} 06^{\prime \prime}, c=90^{\circ} . C / B=0$ beyond this critical angle and $D / B$ as well as $E / B$ are complex. When $b=64^{\circ} 59^{\prime} 30^{\prime \prime}, e=90^{\circ} . E / B=0$ beyond this angle, and only the complex ratio $D / B$ remains. The absolute values of the complex ratios are used in the relative energy calculations.

When the incident wave is transverse, the equation of Blut (2) for the distribution of the incident energy, also given on page 178 of Macelwane (12), is

$$
1=\frac{C^{2} \sin 2 a}{B^{2} \sin 2 b}+\frac{D^{2}}{B^{2}}+\frac{E^{2}}{B^{2}} q \frac{\sin 2 e}{\sin 2 b}+\frac{F^{2}}{B^{2}} q \frac{\sin 2 f}{\sin 2 b} .
$$

The fourth term on the right side represents the relative energy going into the refracted shear wave. Since there is no such wave for Case III, $F=0$ and the expressions for the relative energies are:

$$
\frac{D^{2}}{B^{2}}=\text { relative energy of the reflected shear wave. }
$$

$$
\frac{C^{2} \sin 2 a}{B^{2} \sin 2 b}=\text { relative energy of the reflected longitudinal wave. }
$$

$$
\frac{E^{2}}{B^{2}} q \frac{\sin 2 e}{\sin 2 b}=\text { relative energy of the refracted longitudinal wave. }
$$

The square roots of the relative energy going into the reflected and refracted waves were calculated for the stated values of $b$. Figure 3 shows a plot of them against angle of incidence. Equation [14] was used to check the calculations of Case III.

Knott (10) also studied the case of an incident shear (SV) wave in the mantle against the core. The graph of his results on page 189 of his paper differs noticeably from figure 3 of this work. In figure 3 there is a critical point corresponding to $c=90^{\circ}$ at which the relative energy going into the reflected $\mathrm{P}$ and refracted $\mathrm{P}$ waves becomes zero and the relative energy going into the reflected shear wave becomes one. Under the assumptions of the present calculations this critical point occurs at $b=31^{\circ} 57^{\prime} 06^{\prime \prime}$. In Knott's graph for this case there is no such "zero point." Instead, at approximately $b=31^{\circ}$ in his figure the value of the relative energy of the reflected $P$ wave is zero, of the refracted $P$ wave is 0.82 , and of the reflected $\mathrm{SV}$ wave is 0.17 .

The critical angle of incidence at $b=31^{\circ} 57^{\prime} 06^{\prime \prime}$ in figure 3 may be explained by referring to equations [13a], [13b], and [13c]. Using $a=c=90^{\circ}$ in [13c] gives $D=1.00$. These values of $a$ and $D$ cause equation [13a] to become $E=0$. Equa- 
tion $[13 \mathrm{~b}]$ then becomes $C=\frac{2 \tan 2 b}{1.8897}$. However, even though $C$ is finite, $a=90^{\circ}$ gives zero for the relative energy of the reflected $\mathrm{P}$ wave in equation [15b]. $E=0$ causes the relative energy of the refracted $P$ wave to become zero in equation [15c], and $D=1.00$ causes the relative energy of the reflected $\mathrm{SV}$ wave to become one in equation [15a].

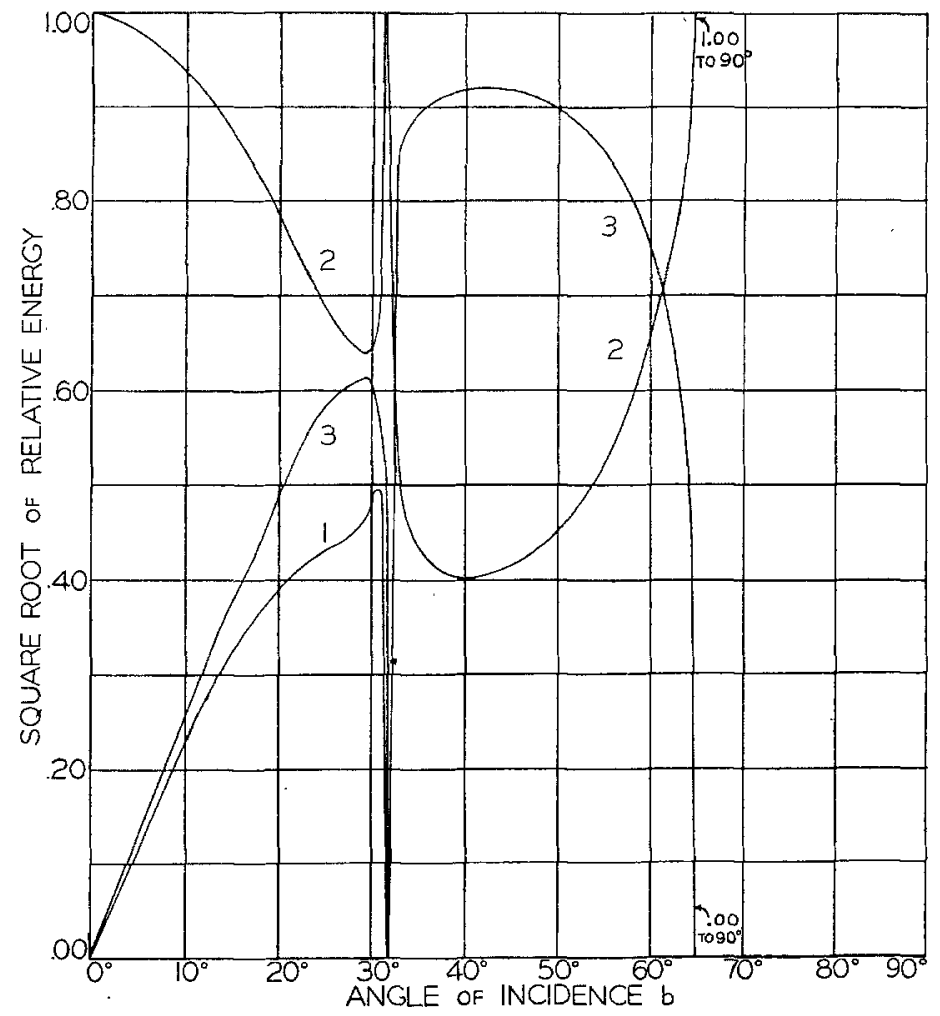

Fig. 3. Incident shear (SV) wave in the mantle against the core.

$1=$ square root of the relative energy of the reflected longitudinal wave.

$2=$ square root of the relative energy of the reflected shear (SV) wave.

$3=$ square root of the relative energy of the refracted longitudinal wave.

There is a second critical angle of incidence in figure 3 . It occurs at $b=64^{\circ} 59^{\prime} 30^{\prime \prime}$. Since $C / B$ is zero for this region of the plot and $e=90^{\circ}$ for this value of $b$, equation [13a] becomes $D=1.00$. Then, using equation [13b], it results that $E=\frac{-2 \sin 2 b}{2.0639}$. However, the fact that $e=90^{\circ}$ causes the relative energy of the refracted $\mathbf{P}$ wave to become zero in equation [15c]. Also, $D=1.00$ in equation [15a] gives one for the relative energy of the reflected SV wave at this value of $b$. This explains the behavior of the relative energies at $b=64^{\circ} 59^{\prime} 30^{\prime \prime}$ in figure 3 .

For Case IV, the incident SH in the mantle against the core, the initial conditions 
are the same as in Case III. Since there is no refracted shear wave, $F=0$ and the tangential strains equation [3a] disappears. Equation [3b] becomes

$$
\text { Tangential stresses, } \quad B=D \text {. }
$$

This equation shows that all of the energy, regardless of the angle of incidence, is going into the reflected $\mathrm{SH}$ wave. Thus the square root of relative energy in this case is always one.

The values of the square roots of relative energy going into the reflected and refracted waves for each of the four cases discussed in this paper may be used in the calculation of the horizontal and vertical components of the displacement produced at the earth's surface by various seismic waves reflected and refracted at the surface of the earth's core. The practical use of such calculations is obvious. They will be described and presented in a future article.

\section{REFERENCES}

1. Berlage, H. P. "Näherungsformeln zur Berechnung der Amplituden der elastischen Wellen, die beim Durchgang einer gegebenen Welle durch eine Unstetigkeitsfläche entstehen," Gerlands Beiträge zur Geophysik, 26:131-140 (1930).

2. Blut, Heinrich. "Ein Beitrag zur Theorie der Reflexion und Brechung elastischer Wellen an Unstetigkeitsflächen," Gerlands Beiträge zur Geophysik, 8:130-144 (1932).

3. Bouasse, H. "Reflexion et refraction," ch. ii in Séismes et sismographes, Delagrave, Paris, pp. 72-89 (1927).

4. Gutenberg, B., and A. Sieberg, "Die Vorläufer bei Fernbeben," Kap. 31 in Geologische, physitalische und angewandte Erdbebenwellen, Fischer, Jena, pp. 284-231 (1923).

5. Gutenberg, B. “Theorie der Erdbebenwellen. Die Bahnen der Erdbebenwellen im Erdinnern," Handbuch der Geophysik, Band 4: Erdbeben, Borntraeger, Berlin, Abschnitt 1:32-80 (1932).

6. Gutenberg, B., and C. F. Richter, "On Seismic Waves" (Fourth Paper), Gerlands Beiträge zur Geophysik, 54:94-136 (1939).

7. Gutenberg, B., ed. Internal Constitution of the Earth [Physics of the Earth-VII], McGrawHill Book Co., New York (1939).

8. Jeffreys, H. "The Reflexion and Refraction of Elastic Waves," Geophysical Supplement, Monthly Notices of the Royal Astronomical Society, 1:321-334 (1926).

9. Knott, C. G. "Reflexion and Refraction of Elastic Waves, with Seismological Applications," Philosophical Magazine, (5) 48:90 ff. (1899).

10. Knott, C. G. "The Propagation of Earthquake Waves through the Earth, and Connected Problems," Proceedings of the Royal Society of Edinburgh, 39 (Pt. II): 157-208 (1919).

11. Macelwane, J. B. "Reflection and Refraction of Earthquake Waves," chap. xii in Seismology [Physics of the Earth-VI], National Research Council of the National Academy of Sciences, Washington, D.C., Bulletin No. 90, pp. 116-120 (1933).

12. Macelwane, J. B., and F. W. Sohon, Introduction to Theoretical Seismology, chap. ii in Part I: Geodynamics, John Wiley and Sons, New York, pp. 147-179 (1936).

13. Wiechert, E. "Ueber Erdbebenwellen. I. Theoretisches über die Ausbreitung der Erdbebenwellen. VIIb. Ueber Reflexion und Durchgang seismischer Wellen durch Unstetigkeitsflächen," Nachrichten d. k. Gesell. Wiss. Göttingen, math.-phys. K1., pp. 66-84 (1919).

Calmformia Institute of Technology

Pasadena, California

(Balch Graduate School of the Geological Sciences, Contribution No. 371) 